\title{
Probing nucleon-nucleon correlations in heavy-ion transfer reactions
}

\author{
S. SZILNER
}

Ruđer Bošković Institute, Zagreb, Croatia

\begin{abstract}
The $\gamma$-particle coincident measurements, performed by coupling of the PRISMA spectrometer to the large $\gamma$ arrays (CLARA and AGATA), demonstrate a strong interplay between single-particle and collective degrees of freedom that is pertinent to the reaction dynamics. By using the unique PRISMA performance in terms of both resolution and efficiency, measurements at very low bombarding energies have been performed. Via transfer of nucleon pairs, valuable information on the component responsible for particle correlations has been derived.
\end{abstract}

\section{Introduction}

The recent revival of transfer reaction studies [1] greatly benefited from the construction of the new generation instrumentation that reached an unprecedented efficiency and selectivity. In the region close to the Coulomb barrier, these reactions reflect a strong interplay between single-particle and collective degrees of freedom and the reaction dynamics [2-4].

Significant progress has been recently achieved by performing studies far below the Coulomb barrier with the PRISMA spectrometer. Transfer cross sections obtained from excitation functions for the closed shell ${ }^{40} \mathrm{Ca}+{ }^{96} \mathrm{Zr}[5]$ and superfluid ${ }^{60} \mathrm{Ni}+{ }^{116} \mathrm{Sn}[6]$ systems have been measured from the Coulomb barrier energy to deep below it. The transfer probabilities have been compared with microscopic calculations that incorporate 
nucleon-nucleon correlations, essential for the population pattern of the single particle levels around the Fermi energy. For the first time in a heavy ion collision, one was able to provide a consistent description of one and two neutron transfer reactions by incorporating, in the reaction mechanism, all known structure information of entrance and exit channels nuclei.

In this work I will focus on the main outcome of the recent studies, critically addressing the new achievements, the present problems and new challenges.

\section{Transfer reaction mechanism}

Transfer reactions have an important impact in the understanding of correlations in the nuclear medium, and play a very important role for the study of the evolution from the quasi-elastic to the deep-inelastic regime [1]. In heavy-ion induced transfer reactions, the constituents of the collision may exchange many nucleons, thus providing information on the contribution of single particle and correlated particle transfers, and on the contribution of surface vibrations (bosons) and their coupling with single particles (fermions). In this sense it is very important to determine the absolute cross sections which can be compared with the state-of-art calculations. From the comparison between data and theory it is possible to evaluate the importance of the elementary modes of excitation in the reaction model.

Total angle and $Q$-value integrated cross sections for multi-neutron and multi-proton channels have been investigated with spectrometers in various systems close to the Coulomb barrier $\left({ }^{58} \mathrm{Ni}+{ }^{208} \mathrm{~Pb}[7],{ }^{40} \mathrm{Ca}+{ }^{208} \mathrm{~Pb}[8]\right.$, ${ }^{40} \mathrm{Ca}+{ }^{96} \mathrm{Zr},{ }^{90} \mathrm{Zr}+{ }^{208} \mathrm{~Pb}[9,10]$, and $\left.{ }^{40} \mathrm{Ar}+{ }^{208} \mathrm{~Pb}[11]\right)$. In these systems one finds that most nuclei produced in transfer reactions have $N / Z$ ratio smaller than one of the compound nucleus, implying the dominance of a direct mechanism in the population of different fragments. For the massive proton transfer channels the isotopic distributions drift toward lower masses, a clear indication that these distributions are affected by evaporation processes. The cross sections for the neutron pick-up drop by almost a constant factor for each transferred neutron, as an independent particle mechanism would suggest.

In all mentioned systems, it has been noticed that the pure proton cross sections display a population of the $(-2 p)$ channel as strong as the $(-1 p)$ one, suggesting the contribution of processes involving the transfer of proton pairs in addition to the successive transfer of single protons. This apparent proton and neutron asymmetric behavior is due to the fact that the one- 
neutron transfer cross section is almost one order of magnitude larger than the one-proton transfer masking the contribution of a pair-transfer mode in the neutron sector by the successive mechanism. As the very short-range pairing interaction redistributes the strength around the pure configurations, it is very important to study the yields distribution of the individual states. This subject will be addressed in next section.

The newly accumulating data are also attracting a renewed interest by theorists. Microscopic models have been used to compute transfer cross sections in light ion induced reactions [12]. Time Dependent Hartree Fock theory has been employed without $[13,14]$ and with [15] approximate inclusion of correlations. Calculations based on Langevin [16] and diffusion equations [17] have been recently performed. Calculations for multinucleon transfer processes have been extensively and successfully performed within semiclassical theories [1,9], in particular by using the GRAZING model [18].

\subsection{Particle vibration couplings}

In the description of the experimental yields, it has been established [1] that the important degrees of freedom in the transfer dynamics are the internal degrees of freedom of the two ions in terms of elementary modes, surface vibrations and single particles. In fact, it is through the excitation of these elementary modes that energy and angular momentum are transferred from the relative motion to the intrinsic degrees of freedom and that mass and charge are exchanged among the two partners. Heavy-ion transfer reactions, thus, should provide the ideal tool for the studies of the particle-vibration coupling scheme. Even if such couplings are essential for the description of many basic states in the vicinity of closed shells, their effects are largely unexplored.

The Ar (neutron transfer channels) [2] and Cl [19] (one proton stripping channels) isotopes have been populated in the ${ }^{40} \mathrm{Ar}+{ }^{208} \mathrm{~Pb}$ reaction. Their $\gamma$ spectra display strong transitions which can be connected with the singleparticle or single-hole states. In addition, through the whole isotopic chain also states that involve combinations of a single particle or hole with a collective boson have been populated. This is illustrated for the Ar isotopic chain in fig. 1. For example, in ${ }^{41} \mathrm{Ar}$, the one-neutron transfer channel, beside the low lying states with a pronounced single-particle character, the decay of the $11 / 2^{-}$state has been observed. This $11 / 2^{-}$state can be understood as a coupling of a collective boson to single-particle states (i.e. $\mid 2^{+},\left(f_{7 / 2}\right)^{1}>$ ) giving a $11 / 2^{-}$stretched configuration. The properties of such states are closely connected with the properties of the vibration quanta, allowing one 


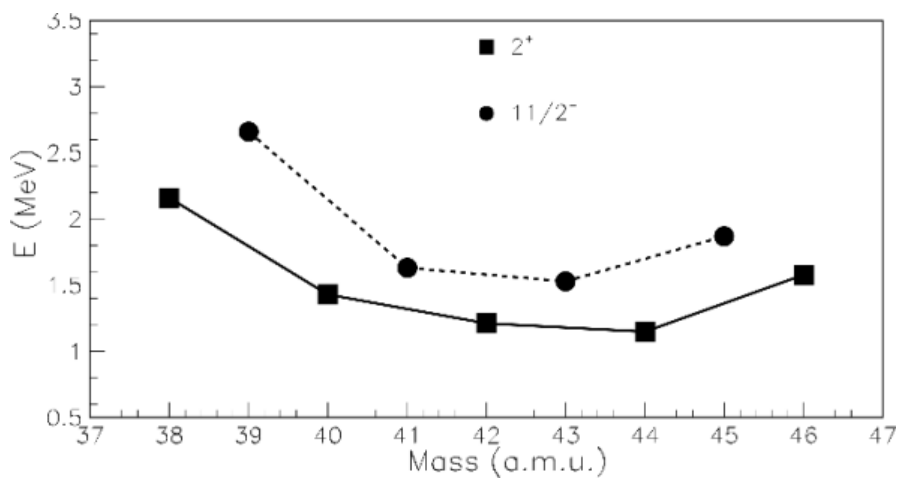

Figure 1: Energies of the $2^{+}$(squares), and 11/2- (circles) states of argon isotopes with $N=20-28$ populated in ${ }^{40} \mathrm{Ar}+{ }^{208} \mathrm{~Pb}$ at $E_{l a b}=255 \mathrm{MeV}$. The energies of $11 / 2^{-}$ in ${ }^{41} \mathrm{Ar}$ and ${ }^{43} \mathrm{Ar}$ are from refs. [2,20]. In the most recent compilation of the ${ }^{45} \mathrm{Ar}$ level properties, a level at $1911(5) \mathrm{keV}$ is a good candidate for the $11 / 2^{-}$state, and was added for completeness.

to follow the development of collectivity in odd isotopic chain, a phenomenon widely discussed in even-even isotopes.

\subsection{Nucleon-nucleon correlations}

With heavy ions multiple transfer of nucleons becomes available in the reaction, giving the possibility to study the relative role of single particle and pair transfer modes. In the sub-barrier domain the two ions probe their mutual interaction only at very large distances, where the nuclear couplings are dominated by transfer processes. The number of open reaction channels is reduced, and the $Q$-value distributions get narrower. These conditions diminish the complexity of coupled channel calculations. Even if the cross sections becomes smaller, the coming into operation of large acceptance magnetic spectrometers made it possible to perform sub-barrier measurements with good ion identification. Transfer yield could be detected at the level of $10^{-4}$ with respect to the elastic channel.

In very recently measured systems one successfully demonstrated the powerful method of using PRISMA [9,21] for such studies, exploiting its unique performance in terms of both resolution and efficiency. Making use of inverse kinematics, target recoils have been detected in multinucleon transfer reactions for the systems ${ }^{96} \mathrm{Zr}+{ }^{40} \mathrm{Ca}[5]$ and ${ }^{116} \mathrm{Sn}+{ }^{60} \mathrm{Ni}[6]$. In both cases the excitation functions at several bombarding energies have been obtained from the Coulomb barrier to 20-25\% below, reaching about $15.5 \mathrm{fm}$ of distance of closest approach. The results of the ${ }^{116} \mathrm{Sn}+{ }^{60} \mathrm{Ni}$ measurement are presented, 


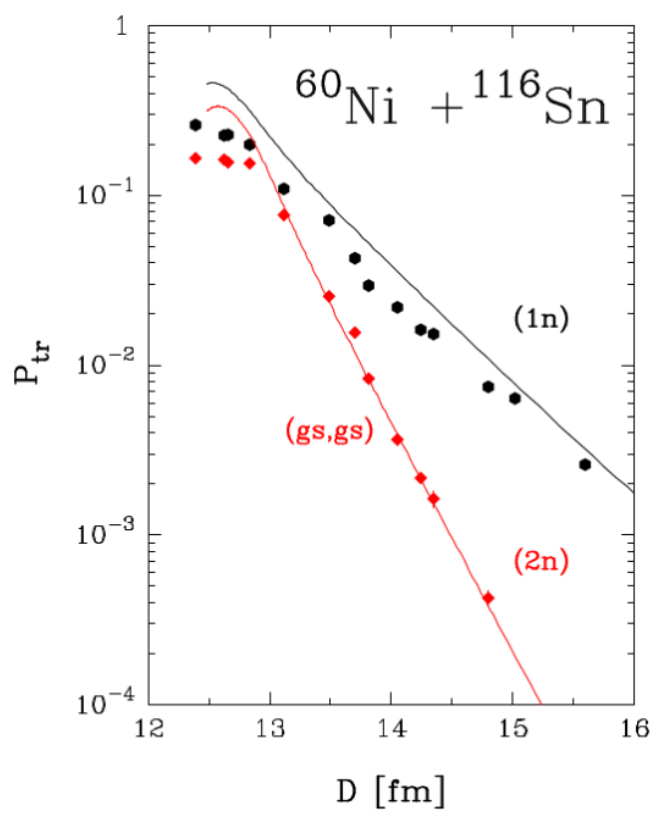

Figure 2: Experimental (points) and microscopically calculated (lines) transfer probabilities $\left(P_{t r}\right.$ defined as the ratio of the transfer cross section to the corresponding Rutherford cross section) as a function of the distance of closest approach $D$ for one- $\left({ }^{61} \mathrm{Ni}\right)$ and two-neutron $\left({ }^{62} \mathrm{Ni}\right)$ transfers in the ${ }^{116} \mathrm{Sn}+{ }^{60} \mathrm{Ni}$ system (from ref. $[6])$.

together with the calculations, in fig. 2 for the $(1 n)$ and $(2 n)$ neutron transfer channels.

To compute the inclusive ( $1 n)$ cross section (full line) one calculated the transfer probability for a given single particle transition and one obtained the total transfer probability by summing over all possible transitions that can be constructed from the single particle states in projectile and target (see ref. [6]), and by weighting each transition with the corresponding spectroscopic factor. The calculations well reproduce the experimental slope and the absolute values. To calculate the $(2 n)$ channel one has to solve the well-known system of semiclassical coupled equations up to the second-order Born approximation. The amplitude consists of three terms, the simultaneous transfer of the pair of nucleons, the nonorthogonality term, and the term which represents the successive process via an intermediate channel. In an independent particle description the first and second term cancel exactly. For the calculation of the $(2 n)$ channel the ground to ground state transition has been included following the formalism of ref. [22]. In this 


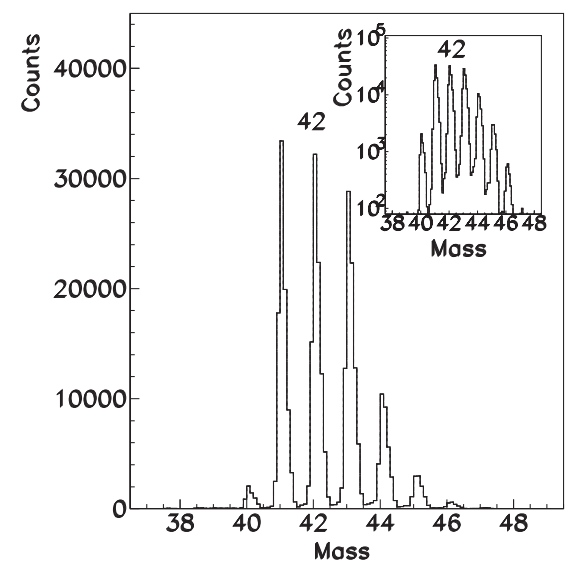

Figure 3: Mass distribution of the $\mathrm{K}$ isotopes obtained in the ${ }^{40} \mathrm{Ar}+{ }^{208} \mathrm{~Pb}$ reaction [11] measured at $\theta_{l a b}=54^{\circ}$ and $E_{l a b}=255 \mathrm{MeV}$. The insert displays the same mass distribution in logarithmic scale.

formalism one can employ the same single particle form factors used for the (1n) channel. The ground states of ${ }^{62} \mathrm{Ni}$ and ${ }^{114} \mathrm{Sn}$ have been described in the BCS approximation.

It is very important to stress that the shown results are not coming from best fit procedure, but that the employed microscopic theory, that incorporates nucleon-nucleon correlations, essential for the population pattern of the single particle levels around the Fermi energy, very well reproduces the experimental data in the whole energy range, in particular, the transfer probability for two neutrons is very well reproduced, in magnitude and slope, by considering solely the ground-ground state transition.

\subsection{Proton-neutron correlations}

In this context, it is also important to investigate the role played by protonneutron correlations. In order to study these correlations one has to use systems where the population of the $( \pm p n)$ channels is allowed by the transfer mechanism ( $Q$-value consideration). These conditions were fulfilled in the ${ }^{40} \mathrm{Ar}+{ }^{208} \mathrm{~Pb}$ system [11], where the angular distributions were studied by PRISMA in a wide angular range (3 different PRISMA angular settings). The total cross sections, obtained from integrating the angular and $Q$-value distributions for the proton striping and neutron pick-up channels concede with the behaviour of previously studied and already discussed systems. The $(+p n)$ channel behaves differently, with the cross section as strong as 
the $(+1 p)$ cross section (see fig. 3). Such behaviour indicates that some additional degrees of freedom have to be considered for that channel.

In heavy-ion reactions the large energy losses may occur, thus we very carefully studied the total kinetic energy loss distributions and the transition from a regime of direct reactions (quasi-elastic) to the more complicated regime of deep inelastic collisions. Even with careful extraction of the quasielastic components only, the obtained cross sections for the $(+p n)$ channel turned out to be enhanced. The strength and presence of the correlation may emerge from a direct comparison of the measured and the microscopically calculated cross sections, where all essential ingredients for accurate description of the nuclear structure and dynamics are employed.

\section{Summary and outlook}

The advent of the large solid angle magnetic spectrometers, coupled to large $\gamma$ arrays, ensured significant advances in the field of multinucleon transfer reactions at energies close to the Coulomb barrier. The detailed studies of the fragments yield distributions and in-beam $\gamma$ emission are important for the understanding of the reaction mechanism and nuclear structure. Via transfer of multiple pairs valuable information on nucleon-nucleon correlations can also be derived. In the comparison between data and theory, the importance of the elementary degrees of freedom, i.e. single particle, phonon and pair modes, can be deduced. It is very important to continue these studies, and to compare the experimental observables with microscopic calculations where nucleon-nucleon correlations are treated together with the relevant structure and dynamics information.

\section{Acknowledgements}

The material presented in this paper is the result of the work of many people which I would like to thank, in particular L. Corradi, T. Mijatović, G. Pollarolo, D. Montanari, E. Fioretto, A. M. Stefanini, J. J. ValienteDobón, G. Montagnoli, F. Scarlassara, C. A. Ur, and A. Goasduff. This work was partly supported by the EC FP7 ENSAR (262010), by Croatian Science Foundation (project 7194), and by the Scientific center of excellence for advance materials and sensors. 


\section{References}

[1] L. Corradi, G. Pollarolo, and S. Szilner, J. of Phys. G 36 (2009) 113101.

[2] S. Szilner et al., Phys. Rev. C 84 (2011) 014325.

[3] S. Lunardi et al., Phys. Rev. C 76 (2007) 034303.

[4] J. J. Valiente Dobón, this proceeding.

[5] L. Corradi et al., Phys. Rev. C 84 (2011) 034603.

[6] D. Montanari et al., Phys. Rev. Lett 113 (2014) 052501.

[7] L. Corradi et al., Phys. Rev. C 66 (2002) 024606.

[8] S. Szilner et al., Phys. Rev. C 71 (2005) 044610.

[9] S. Szilner et al., Phys. Rev. C 74 (2007) 024604.

[10] M. Varga Pajtler et al., Nucl. Phys. A 941 (2015) 273.

[11] T. Mijatović, Ph.D. thesis, Faculty of Science, University of Zagreb (2015).

[12] G. Potel, F. Barranco, E. Vigezzi, and R. A. Broglia, Phys. Rev. Lett. 105 (2010) 172502.

[13] C. Simenel, Phys. Rev. Lett. 105 (2010) 192701.

[14] K. Sekizawa, and K. Yabana, Phys. Rev. C 88 (2013) 014614.

[15] G. Scamps, and D. Lacroix, Phys. Rev. C 87 (2013) 014605.

[16] V. Zagrebaev, and W. Greiner, Phys. Rev. Lett. 101 (2008) 122701.

[17] V. V. Sargsyan et al., Phys. Rev. C 88 (2013) 064601.

[18] A. Winther, Nucl. Phys. A 572, 191 (1994); Nucl. Phys. A 594 (1995) 203.

[19] S. Szilner et al., Phys. Rev. C 87 (2013) 054322.

[20] D. Mengoni et al., Phys. Rev. C 82 (2010) 024308.

[21] D. Montanari et al., Eur. Phys. J. A 47 (2011) 4.

[22] J. H. Sorensen and A. Winther, Nucl. Phys. A 550 (1992) 306. 